\title{
Toxicité pulmonaire des médicaments : ce que le réanimateur doit connaître?
}

\author{
Drug-Induced Lung Toxicities: What the Intensivist Should Know?
}

\author{
A. Parrot - A. Gibelin $\cdot$ T. Issoufaly $\cdot$ G. Voiriot $\cdot$ M. Djibré $\cdot$ J.M. Naccache $\cdot$ J. Cadranel $\cdot$ M. Fartoukh \\ Reçu le 25 septembre 2017; accepté le 11 décembre 2017 \\ (C) SRLF et Lavoisier SAS 2018
}

Résumé Le diagnostic d'insuffisance respiratoire aiguë d'origine médicamenteuse est un véritable défi. Les traitements à l'origine d'une toxicité pulmonaire sont très nombreux. Les nouveaux traitements, notamment en oncohématologie (thérapie ciblée, immunothérapie), ne sont pas en reste. L'expression clinique très hétérogène est sous-tendue par des mécanismes lésionnels très différents. Après avoir écarté une infection ou un œdème pulmonaire, il faut savoir évoquer une pneumopathie médicamenteuse. La réalisation d'un scanner thoracique et d'une fibroscopie bronchique avec lavage bronchoalvéolaire peut être utile pour exclure une pathologie infectieuse et suggérer une pathologie médicamenteuse. Le traitement est l'arrêt du médicament et repose fréquemment dans ces formes graves sur une corticothérapie.

Mots clés Détresse respiratoire · Pneumopathie médicamenteuse $\cdot$ Toxicité pulmonaire

\begin{abstract}
Acute respiratory failure related to drug toxicity is challenging. Several drugs, also including targeted therapy and immunotherapy, have been reported to induce lung toxicity. Clinical and pathological presentations are heterogeneous suggesting different toxicity pulmonary mechanisms. Infection or pulmonary edema should be ruled out, and then drug-induced pneumonitis diagnosis should be considered. CT scan and bronchoscopy with bronchoalveolar lavage are
\end{abstract}

\footnotetext{
A. Parrot $(\bowtie) \cdot$ T. Issoufaly $\cdot$ J.M. Naccache $\cdot$ J. Cadranel

Service de pneumologie, AP-HP,

hôpitaux universitaires de l'Est-Parisien,

Pôle TVAR, hôpital Tenon, 75020 Paris

e-mail : antoine.parrot@aphp.fr

A. Gibelin · G. Voiriot · M. Djibré · M. Fartoukh

Service de réanimation et USC médico-chirurgicale,

AP-HP, hôpitaux universitaires de l'Est-Parisien,

Pôle TVAR, hôpital Tenon, 75020 Paris

A. Gibelin · J. Cadranel $\cdot$ M. Fartoukh

UPMC, université Paris-VI, 75006 Paris, France
}

helpful for the disease diagnosis. Due to the severity, the identified drug should be stopped and more frequently steroids should be started.

Keywords Respiratory failure · Drug-induced pneumonitis · Lung toxicity

\section{Introduction}

De nombreux médicaments sont responsables d'une toxicité pulmonaire conduisant à une insuffisance respiratoire aiguë (IRA). Leur incidence est mal évaluée et largement sous-estimée. Le nombre d'admissions hospitalières en relation avec une toxicité médicamenteuse pulmonaire est en augmentation constante ( $+140 \%$ en 10 ans) même si cette variation importante est en partie liée à une meilleure sensibilisation au diagnostic, et il est probable qu'il ne cessera d'augmenter avec le vieillissement de la population [1-4]. En réanimation, en situation de syndrome de détresse respiratoire aiguë (SDRA) selon la classification de Berlin, l'incidence des pneumopathies médicamenteuses (PM) varie entre 2 et $10 \%[5,6]$. Dans le sous-groupe des SDRA, sans les facteurs de risque classique de Berlin (environ $10 \%$ des SDRA) ou des pneumopathies infiltrantes diffuses aiguës, la fréquence des PM varie entre 17 et $28 \%$ [6-8].

La mortalité des IRA médicamenteuses est très élevée, entre 40 et $70 \%$ [5-7]. Les thérapies ciblées en oncologie, malgré leur «nom enchanteur », en cas de toxicité pulmonaire, induisent toujours une forte mortalité de l'ordre de $50 \%$ [9]. Poser le diagnostic d'insuffisance respiratoire aiguë d'origine médicamenteuse (IRAM) est donc indispensable, mais il est difficile pour les raisons suivantes :

- la liste des médicaments susceptibles de se compliquer d'IRAM s'étoffe régulièrement (Tableaux 1-3) [3,10-15]. Mais tous les médicaments ne sont pas responsables d'IRA avec la même fréquence ; 
- le spectre clinique des IRAM est large, allant de l'atteinte du parenchyme la plus fréquente (pneumopathie interstitielle diffuse, SDRA, hémorragie intra-alvéolaire, pneumopathie à éosinophile), en passant par celle des voies aériennes supérieures ou inférieures (bronchospasme), ou centré sur les vaisseaux pulmonaires (hypertension pulmonaire, hémoptysie). En outre, un même médicament n'est pas nécessairement associé au même type d'atteinte $[3,15,16]$, par exemple le nivolumab est responsable de SDRA ou de pneumonie organisée $[17,18]$;

- les patients reçoivent souvent une combinaison de traitements, qui isolément ne sont pas nécessairement responsables de PM, mais dont l'association est toxique [5,12] ;

- la relation temporelle avec le médicament n'est pas toujours évidente, remontant parfois à plusieurs années ;

- le délai de réflexion est réduit, le pronostic vital étant engagé ;

- la balance bénéfice/risque des investigations à entreprendre pour obtenir le diagnostic est difficile à évaluer ;

- il est le plus souvent impossible d'incriminer un médicament avec certitude dans la mesure où la sévérité de l'IRA rend irréalisable la pratique d'un test de réintroduction. Un bon argument est la régression après arrêt du traitement ;

Tableau 1 Pneumopathie médicamenteuse s'accompagnant d'IRA en oncohématologie

\begin{tabular}{|c|c|}
\hline \multirow[t]{2}{*}{ Méthotrexate** } & Oxaliplatine \\
\hline & Idélalisib \\
\hline Bléomycine** & Évérolimus* \\
\hline Vinorelbine & Cétuximab \\
\hline Cytosine arabinoside* & $\begin{array}{l}\text { Inhibiteurs des tyrosines } \\
\text { kinases** }\end{array}$ \\
\hline Gemcitabine* & $\begin{array}{l}\text { Inhibiteurs des points } \\
\text { de contrôle de l'immunité** }\end{array}$ \\
\hline Mitomycine $\mathrm{C}^{*}$ & Rituximab* \\
\hline BCNU* & Trastuzumab \\
\hline Cyclophosphamide* & $\begin{array}{l}\text { Thalidomide, Lénalidomide } \\
\text { Bortézomib }\end{array}$ \\
\hline Chlorambucil & Fludarabine, cladribine \\
\hline \multicolumn{2}{|l|}{ Docétaxel } \\
\hline \multicolumn{2}{|l|}{ Paclitaxel } \\
\hline \multicolumn{2}{|c|}{ Acide tout transrétinoïque* } \\
\hline \multicolumn{2}{|l|}{ Nilutamide* } \\
\hline \multicolumn{2}{|c|}{ Transfusions de plaquettes } \\
\hline \multicolumn{2}{|l|}{ Facteurs de croissance } \\
\hline \multicolumn{2}{|l|}{$(\mathrm{G}-\mathrm{CSF})^{*}$} \\
\hline \multicolumn{2}{|c|}{$\begin{array}{l}\text { - Pneumopathies s'accompagnant d'IRA }\left(\mathrm{PaO}_{2}<60 \mathrm{mmHg}\right) \\
\text { constituées en moins de } 2 \text { mois. }\end{array}$} \\
\hline
\end{tabular}

* Plus de 10 cas rapportés ; ** plus de 100 cas rapportés
- l'arrêt d'un traitement présumé pneumotoxique par erreur peut être dramatique en cancérologie, en l'absence de traitement alternatif [19].

Les IRA résultant d'un mécanisme indirect par atteinte du système ventilatoire actif (centres nerveux et muscles respiratoires), d'un effet immunosuppresseur source d'infections opportunistes, de l'usage de stupéfiants ou d'une intoxication aiguë volontaire, ne seront pas abordées. L'IRAM résultant de la seule atteinte pleurale est rare [20].

Pour aborder ces IRAM nous répondrons aux questions suivantes :

- quels patients?

- quels médicaments?

- quels tableaux cliniques?

- quelle stratégie?

- quel traitement?

\section{Quels patients ?}

En l'état actuel des connaissances, tout patient est susceptible de développer une complication pulmonaire d'origine médicamenteuse. Il n'est pas possible de prédire la survenue d'une toxicité pulmonaire pour un individu donné. Néanmoins, on peut repérer des facteurs favorisants qui peuvent s'associer. Ainsi, un âge avancé favorise la toxicité pulmonaire de la bléomycine. Le polymorphisme génétique joue certainement un rôle, comme cela est décrit pour la coumadine et le méthotrexate, mais l'impact clinique est très réduit. Certaines ethnies sont plus exposées à une toxicité pulmonaire : le risque de pneumopathie au gefitinib est de $2 \%$ au Japon contre $0,3 \%$ aux États-Unis [2,21]. L'état pulmonaire préexistant (pneumopathie interstitielle diffuse, pneumonectomie, cancer pulmonaire) est susceptible de favoriser l'apparition d'une PM, comme cela est décrit pour le méthotrexate chez les patients atteints d'une polyarthrite rhumatoïde, l'amiodarone, les inhibiteurs des tyrosines kinases et les inhibiteurs des points de contrôle immunitaire [12,22,23]. Pour ces derniers, il existe un risque accru de PM lorsqu'ils sont administrés pour un cancer pulmonaire comparé à un mélanome. L'oxygène accroît probablement la toxicité d'une chimiothérapie concomitante ou antérieurement administrée, et celle de l'amiodarone. L'atopie joue probablement un rôle. L'association de traitements cytotoxiques comme le BCNU ou d'inhibiteurs des points de contrôle immunitaire augmente le risque de PM [24,25]. Une radiothérapie antérieure, alors qu'elle n'a entraîné aucune atteinte pulmonaire initiale, peut favoriser l'apparition d'une pneumopathie radique dans les territoires irradiés à l'occasion d'une nouvelle chimiothérapie, notamment par les taxanes, ou la survenue d'une hémoptysie en cas de traitement par bévacizumab. 


\begin{tabular}{|c|c|c|}
\hline Nitrofurantoïne** & Amiodarone** & Rapamycine \\
\hline Cotrimoxazole* & Hydrochlorothiazide & Azathioprine \\
\hline Extencilline & Tocaïnide & Ciclosporine \\
\hline Ampicilline, Amoxicilline & Captopril & \\
\hline Céfalexine & Perindopril & Phénytoïne \\
\hline \multirow[t]{2}{*}{ Latomoxef } & Propanolol* & Carbamazepine* \\
\hline & & Acide valproïque \\
\hline Ceftriaxone & Métipranolol & Halopéridol \\
\hline \multicolumn{3}{|l|}{ Céfotaxime } \\
\hline Ceftazidime & Céliprolol & Bromocriptine \\
\hline Ceftaroline & Aténolol & Maprotiline \\
\hline Érythromycine & Nifédipine & \\
\hline Tétracycline & Héparine & \\
\hline \multirow[t]{3}{*}{ Minocycline } & Antivitamine-K & \\
\hline & Streptokinase & \\
\hline & Abciximab & \\
\hline Daptomycine* & & Aspirine \\
\hline Éthambutol & D-Pénicillamine* & Ibuprofène \\
\hline Salazosulfapyridine* & Méthotrexate & Diclofénac \\
\hline \multicolumn{3}{|l|}{ Mésalazine } \\
\hline & Abatacept & Diflunisal \\
\hline & Étanercept & \\
\hline & Infliximab & \\
\hline & Léflunomide & \\
\hline & Cyclophosphamide & \\
\hline Acétaminophène & Propylthiouracile* & Déféroxamine mésylate \\
\hline Paracetamol & Interféron alpha* & Insuline ? \\
\hline Lidocaïne & Tocolytiques* & Prostacycline \\
\hline Buprénorphine & & «Produits sanguins labiles» \\
\hline \multicolumn{3}{|l|}{ Naloxone* } \\
\hline Fentanyl & & \\
\hline
\end{tabular}

\section{Quels médicaments ?}

Un adage simple est que tout médicament peut être à l'origine d'une IRAM. En effet, en milieu oncohématologique, des IRAM ont été rapportées avec la quasi-totalité des médicaments cytotoxiques (Tableau 1). De ce fait, la majorité des patients traités pour une hémopathie ou une tumeur solide sont des candidats potentiels à de telles complications. Certains médicaments comme le méthotrexate, la bléomycine, la cytosine arabinoside sont fréquemment incriminés. Les thérapies ciblées, notamment les inhibiteurs des tyrosines kinases, et l'immunothérapie, en dépit de leur mécanisme d'action plus spécifique sur la tumeur, sont des sources de pneumopathies graves $[14,18,26]$.

En milieu non oncohématologique, la liste des médicaments incriminés (Tableau 2) est extrêmement longue. Des IRAM s'observent aussi bien chez les patients immunodé- primés (sida, traitements immunosuppresseurs) que chez les patients recevant des médicaments à visée anti-infectieuse, anti-inflammatoire ou antalgique, des traitements cardiotropes, rhumatologiques, ou prenant une prophylaxie antiinfectieuse à l'occasion d'un voyage en pays tropical, ou pris en charge en milieu anesthésique.

Ces listes ne sont pas exhaustives, Pubmed et Pneumotox (www.pneumotox.com) permettent de se tenir à jour. Dans cette mise au point, nous inclurons dans ces listes de médicaments, par abus de langage, les produits sanguins labiles.

Les médicaments responsables d'atteintes pulmonaires le sont, quelle que soit la voie d'administration, comme l'illustrent les pneumopathies au méthotrexate décrites après administration par voie orale, intraveineuse ou intrathécale, les pneumopathies à la bléomycine administrée par voie intraveineuse ou intrapleurale ou les pneumopathies au métipranolol après instillation intraoculaire. 


\begin{tabular}{|ll|}
\hline $\begin{array}{l}\text { Tableau } 3 \\
\text { naire d'origine médicamenteuse s'accompagnant d'IRA }\end{array}$ \\
\hline En oncohématologie & En dehors de \\
& l'oncohématologie \\
\hline Bronchospasme & \\
Mitomycine & AINS \\
Vindésine & Inhibiteurs de l'enzyme de \\
& conversion \\
Vinorelbine & Bêtabloquants \\
Vinblastine & Pentamidine \\
Gemcitabine & Colimycine \\
Matuzamab & \\
Rituximab & \\
Bronchiolite oblitérante & \\
Busulfan & D-pénicillamine \\
Hypertension artérielle pulmonaire \\
Agents alkylants & Fenfluramine \\
(Carmustine, & \\
Cyclophosphamide, & \\
Mitomycine) & Bêtabloquants \\
Bléomycine & \\
Gemcitabine & \\
Dasatinib & \\
\end{tabular}

Les mécanismes de la toxicité pulmonaire sont multiples, complexes et mal élucidés [27]. Classiquement sont décrits ceux en rapport avec :

- une dose toxique suite à des prises répétées ou par un phénomène d'idiosyncrasie (par exemple anomalie génétique responsable d'une incapacité à métaboliser le médicament qui fait que ce dernier devient toxique). Il en va ainsi de la majorité des pneumopathies au busulfan, au BCNU (carmustine), à la bléomycine et à l'amiodarone. Mais on rapporte aussi des pneumopathies hypoxémiantes à la bléomycine avec de faibles doses de bléomycine ou des durées d'administration courtes ;

- une hypersensibilité suggérée par la notion de manifestations respiratoires antérieures, une alvéolite lymphocytaire (ou à éosinophile). Le meilleur exemple est le méthotrexate. Ce type de mécanisme en principe n'autorise pas une réintroduction avec le même médicament.

Parfois, ils résultent d'une association entre mécanisme toxique et mécanisme d'hypersensibilité, par exemple les pneumopathies radiques : en effet, les lésions pulmonaires sont dose-dépendantes, mais la présence d'une alvéolite lymphocytaire du côté controlatéral à l'irradiation suggère un mécanisme d'hypersensibilité retardée.

Plus rarement, les médicaments induisent des réactions immunologiques complexes avec des tableaux d'hémorragie intra-alvéolaire (propylthiouracile), de lupus et d'hypersen- sibilité médicamenteuse (en anglais décrit sous le nom de drug reaction with eosinophilia and systemic symptoms).

Pour les thérapies ciblées, le gefitinib agirait sur des mécanismes de régénération (réparation) de l'épithélium alvéolaire favorisant la survenue de PM [14,28], le dasatinib ciblerait la voie du PDGF (Platelet Derived Growth Factor) favorisant la survenue d'une hypertension artérielle pulmonaire $[10,11]$. La toxicité des inhibiteurs des points de contrôle immunitaire résulte possiblement d'une réaction exagérée de l'auto-immunité.

\section{Quels tableaux cliniques?}

\section{Atteinte des voies aériennes supérieures}

L'obstruction des voies aériennes supérieures peut être en rapport avec un angio-œdème. Cliniquement, il s'agit d'une IRA qui s'installe en une dizaine d'heures, caractérisée par une dyspnée inspiratoire associée à un œdème des lèvres, de la langue, une odynophagie, un stridor et des troubles de la salivation, pouvant conduire à l'intubation. La cause la plus fréquente est l'introduction récente d'inhibiteurs de l'enzyme de conversion de l'angiotensine, mais des cas sont rapportés après plusieurs années de traitement.

D'autres médicaments peuvent être responsables d'une IRA par obstruction des voies aériennes supérieures, relevant de différents mécanismes : anaphylaxie (aspirine, pénicilline) ; compression par hématomes sous anticoagulants.

\section{Atteinte des bronches}

Les manifestations bronchiques médicamenteuses peuvent avoir les expressions suivantes : fréquemment bronchospasme réversible ; plus rarement bronchiolite oblitérante irréversible, ou hémoptysie.

\section{Bronchospasme}

Cliniquement, le tableau se traduit par une IRA survenant quelques minutes à quelques heures après la prise médicamenteuse, associant des sibilants et parfois des signes systémiques : hypotension, signes cutanés (rash, urticaire, angioœdème), fièvre [3]. La radiographie thoracique est normale ou inchangée. Le diagnostic est suspecté dans les circonstances suivantes : un aérosol d'antibiotiques en cours (colimycine, pentamidine...) ; une perfusion d'alcaloïdes de la pervenche, notamment en cas d'association avec de la mitomycine, de gemcitabine ou d'anticorps monoclonaux humanisés (matuzumab, trastuzumab, rituximab) $[3,10]$; une prise récente de bêtabloquants, d'inhibiteurs de l'enzyme de conversion ou d'anti-inflammatoires non stéroïdiens responsables dans près de $8 \%$ des cas de l'aggravation de l'asthme 
dans une série de patients asthmatiques ventilés. En anesthésie, devant un bronchospasme inattendu, il faut évoquer une allergie aux curares, aux anesthésiques locaux, voire au latex ou à l'antibioprophylaxie [29]. Le traitement est essentiellement symptomatique avec l'arrêt de la perfusion, si elle est en cours. La récidive est parfois prévenue par les bronchodilatateurs et les corticoïdes.

\section{Bronchiolite oblitérante}

Décrite avec la D-pénicillamine, le busulfan, la bronchiolite oblitérante se traduit par une dyspnée croissante sur quelques mois, associée à un couinement inspiratoire (squeak). La radiographie thoracique est normale, le scanner thoracique met en évidence une perfusion en mosaïque avec un piégeage expiratoire, et l'étude de la fonction respiratoire objective un syndrome obstructif. Le plus souvent, l'évolution est irréversible, malgré l'administration d'une corticothérapie.

\section{Hémoptysie}

Au cours des premiers essais de traitement des cancers bronchiques non à petites cellules par bévacizumab, des hémoptysies fatales ont été rapportées, survenant particulièrement en cas de cancers épidermoïdes de localisation centrale conduisant à une contre-indication de cette molécule dans ce type histologique [30]. Actuellement, cette complication survient chez moins de $1 \%$ des patients recevant ce traitement pour un adénocarcinome pulmonaire favorisé par une radiothérapie en cours. Récemment, il a été rapporté une hémoptysie fatale après un traitement par crizotinib pour un cancer pulmonaire associé à un réarrangement du gène $A L K$.

\section{Atteinte vasculaire avec hypertension pulmonaire}

Le tableau clinique d'une hypertension artérielle pulmonaire iatrogène ne diffère en rien de celui d'une hypertension pulmonaire. La recherche d'une cause médicamenteuse fait partie de l'enquête étiologique. L'hypertension artérielle pulmonaire iatrogène s'observe, après une chimiothérapie telle que le dasatinib [31], dans le cadre d'une maladie veino-occlusive après la prise d'agents alkylants [32], de gemcitabine [33], ou d'une microangiopathie thrombotique avec atteinte rénale rapportée avec la mitomycine, ou après prise de fenfluramine et de benfluorex [11]. L'évolution est souvent mortelle à court terme ; elle peut cependant être favorable après l'arrêt du dasatinib.

\section{Atteinte parenchymateuse}

\section{Pneumopathies alvéolaires suraiguës}

L'insuffisance respiratoire suraiguë s'installe en quelques minutes ou quelques heures. L'hypoxémie est ici majeure, imposant parfois le recours à la ventilation invasive. La pneumopathie peut s'accompagner d'une fièvre élevée ou d'une hypotension, s'intégrant parfois dans un syndrome de fuite capillaire pulmonaire.

Ce tableau a été rapporté :

- au cours ou au décours d'une perfusion de vinorelbine, d'amphotéricine B liposomale trop rapide source d'un œdème hémodynamique, d'une transfusion de plaquettes de rituximab [16] ou de trastuzumab ;

- après la réintroduction accidentelle d'un médicament ayant déjà entraîné une allergie :

- en milieu oncologique : le méthotrexate (éventuellement après une première alerte ou par voie intrathécale : le mécanisme suspecté étant alors un œdème neurogénique), le cyclophosphamide à l'origine d'une réaction anaphylactique sans œdème de Quincke ni urticaire, la vinblastine ;

- en milieu non oncohématologique : le cotrimoxazole, l'hydrochlorothiazide, l'halopéridol, la nitrofurantoïne, la pyriméthamine, le périndopril ou le propranolol ;

- après un geste particulier : anesthésie locale à la lidocaïne, recours à la naloxone en salle de réveil, talcage pleural, perfusion d'abciximab encadrant une angioplastie avec pose d'un stent, angiographie ou chirurgie pulmonaire chez un malade traité par amiodarone, injection épidurale de fentanyl ou administration de produits sanguins labiles (culots globulaires, plaquettes, plasma frais congelé) ou non (gammaglobulines). En principe, cet accident survient dans les six heures suivant le début de la transfusion;

- lors de recours à une thérapeutique susceptible d'engendrer un œdème pulmonaire par un mécanisme plus ou moins élucidé : nifédipine, buprénorphine ou perfusion de prostacycline en cas d'hypertension pulmonaire liée à une maladie veino-occlusive ou une hémangiomatose capillaire.

\section{Pneumopathies alvéolaires aiguës}

Plus souvent, il s'agit d'une pneumopathie alvéolaire développée en 24 à 72 heures, correspondant soit à un œdème pulmonaire, soit à une hémorragie intra-alvéolaire.

\section{Cdème pulmonaire non cardiogénique}

Cliniquement, il s'agit d'un SDRA, parfois associé à un syndrome de fuite capillaire pulmonaire avec une baisse des protides. L'histologie correspond à un dommage alvéolaire diffus. Le diagnostic doit être évoqué si les facteurs de risque classiques du SDRA ne sont pas présents. Ce tableau est rapporté après : 
- des gestes particuliers :

- en milieu oncologique : survenue de l'IRA en postopératoire $\left(\mathrm{FiO}_{2}\right.$ élevée), chez des patients recevant de la bléomycine. La fréquence de survenue de cette atteinte est très faible $(<2 \%)$;

- en milieu non oncohématologique : talcage pleural, injections de silicone ;

- des situations particulières :

- en milieu oncologique : baisse précoce de corticoïdes administrés pour une atteinte pulmonaire non hypoxémiante attribuée à la bléomycine, au melphalan ou à la mitomycine ; insuffisance rénale chronique chez un patient recevant de la bléomycine par voie intrapleurale ; sortie accélérée et massive de globules blancs avec le granulocyte-colony-stimulating factor (GCSF) au décours d'une neutropénie induite en l'absence de pneumonie préalable tout en sachant que cette toxicité est très discutée ;

- en milieu non oncohématologique : administration de cordarone après une chirurgie ou ventilation avec une haute concentration d'oxygène ; administration de tocolytiques,

- des traitements particuliers :

- en milieu oncologique : le méthotréxate, le BCNU, la bléomycine, l'acide tout transrétinoïque (dans ce cas, l'atteinte parenchymateuse peut s'accompagner d'une atteinte des séreuses - plèvre et péricarde - d'œdèmes, d'une prise de poids, d'une hypotension et d'une insuffisance rénale) et plus récemment le gefitinib [34,35], le crizotinib [36,37], la gemcitabine [3] et le nivolumab [18] ;

- en milieu non oncohématologique : le méthotrexate, l'ibuprofène, l'aténolol, l'intoxication chronique à l'aspirine.

\section{Hémorragie intra-alvéolaire}

Histologiquement, l'hémorragie intra-alvéolaire est en rapport avec trois types de lésions : la capillarite pulmonaire ; des lésions aspécifiques (décrites sous le terme de bland pulmonary hemorrhage dans la littérature anglo-saxonne) notamment au cours de surdosage en anticoagulants, après fibrinolytiques d'autant qu'il existe une hyperpression veineuse pulmonaire, ou de traitement par la cordarone ; et le dommage alvéolaire diffus.

L'hémoptysie associée à l'IRA suggère le diagnostic, mais elle n'est pas toujours présente. De nombreux traitements sont impliqués : la D-pénicillamine, un traitement anticoagulant, fibrinolytique, ou antiagrégant plaquettaire d'autant plus que ces traitements sont associés entre eux [38], le moxalactam, la nitrofurantoïne, l'amiodarone, le propylthiouracile, la phénytoïne, l'acide valproïque ou l'azathioprine, certaines chimio- thérapies (gemcitabine, pemetrexed, évérolimus, rituximab, bévacizumab, crizotinib, bortézomib) [39,40]. L'hémorragie intra-alvéolaire peut également s'observer après injection de silicone. Parfois, l'hémorragie intra-alvéolaire revêt l'aspect d'une maladie de système associée à des signes rénaux ou à des lésions cutanées, d'un purpura thrombopénique, ou est associée à un syndrome hémorragique diffus.

\section{Pneumopathies interstitielles aiguës et subaiguës}

Situations les plus fréquentes, il s'agit d'une insuffisance respiratoire aiguë (survenant en 1 à $10 \mathrm{j}$ ) ou subaiguë (10 jours à 2 mois). La fièvre est fréquente [14]. Les rashs ou l'éosinophilie sanguine sont plutôt rapportés en milieu non oncohématologique. L'imagerie thoracique révèle des opacités bilatérales, le plus souvent du verre dépoli, mais il peut y avoir des condensations alvéolaires, parfois initialement asymétriques, s'accompagnant exceptionnellement d'adénopathies médiastinales ou d'atteinte pleurale.

En milieu oncologique, les pneumopathies interstitielles aiguës ou subaiguës sont observées avec les chimiothérapies (le méthotrexate, le melphalan, la bléomycine, le cyclophosphamide, la gemcitabine, la carmustine, le docétaxel, la mitomycine, le chlorambucil, le nilutamide, l'hydroxyurée, l'idélalisib), un grand nombre inhibiteur des tyrosines kinases (gefitinib, sorafénib, sunitinib, erlotinib, crizotinib...) [9,14,22,36], les inhibiteurs des points de contrôle de l'immunité (nivolumab...) [12,18], les anticorps monoclonaux (rituximab [près de $4 \%$ des patients traités pour un lymphome non hodgkinien] [41], le trastuzumab), et les inhibiteurs de la voie de signalisation mTOR [15]. Les pneumopathies radiques, apparaissant généralement trois semaines à six mois après la fin de l'irradiation, répondent à ce cadre sémiologique, mais s'en individualisent par des lésions très focalisées aux champs d'irradiation. Il est également décrit les pneumonies radiques de rappel (radiation recall pneumonitis), survenant habituellement en territoire irradié, déclenchées par l'administration ultérieure d'une chimiothérapie, mais en général l'atteinte est peu hypoxémiante.

En dehors de la chimiothérapie, les médicaments en cause sont très divers : ampicilline, extencilline, cyclines, céphalosporines, nitrofurantoïne, ibuprofène, salazosulfapyridine, étanercept, méthotrexate, cyclophosphamide, rapamycine, carbamazépine, phénytoöne, déféroxamine, amiodarone, chloroquine, acide valprö̈que, bromocriptine, maproptiline, propylthiouracile, dothiépine, périndopril, diclofénac, paracétamol ou interféron alpha, etc.

\section{Pneumonies organisées}

Des tableaux de pneumonies organisées évoluant vers l'IRA avec recours à la ventilation artificielle sont également rapportés $[42,43]$. Ils sont soit isolés avec la prise régulière de 
nitrofurantoïne, ou après irradiation pour cancer du sein, dans un délai de 15 jours à deux mois, et caractérisés par des opacités alvéolaires migratrices, soit associés à une sémiologie plus complexe comprenant des signes cutanés, un profil septique-like ou une anémie hémolytique (phénytoïne).

\section{Quelle stratégie ?}

L'IRA induite par un traitement est rare. Il faut écarter en priorité une infection, un œdème aigu cardiogénique ou une embolie pulmonaire, et il s'agit par conséquent d'un diagnostic d'exclusion [2].

En pratique, dans notre expérience, nous procédons de la manière suivante :

\section{Analyse sémiologique clinique, biologique et radiologique}

Elle permet de définir la composante majeure de l'IRA, et de centrer la discussion sur les diagnostics prioritaires. Devant une IRA, il faut commencer par identifier le type d'atteinte :

- une atteinte de voies aériennes supérieures : dyspnée inspiratoire, stridor, œdème de langue sont les éléments du diagnostic. Il s'agit d'une des rares situations où le diagnostic prioritaire à évoquer est une étiologie médicamenteuse ;

- une atteinte des voies aériennes inférieures avec un bronchospasme. Les diagnostics prioritaires sont une exacerbation d'une maladie bronchique (bronchopneumopathie chronique obstructive [BPCO], asthme) ou un œdème du poumon. Si ces deux diagnostics ne paraissent pas compatibles, un bronchospasme d'origine médicamenteuse peut être une piste, d'autant que l'événement est inattendu, qu'une chimiothérapie ou un aérosol est en cours ;

- une atteinte vasculaire. Le tableau clinique d'une hypertension artérielle pulmonaire iatrogène est celui d'une hypertension pulmonaire. Après avoir écarté une hypertension pulmonaire secondaire (maladie cardiaque, maladie pulmonaire chronique, embolie pulmonaire), la recherche d'une cause médicamenteuse fait partie de l'enquête étiologique d'une hypertension artérielle pulmonaire ;

- une atteinte du parenchyme. La discussion diagnostique est plus nuancée. En principe, les deux diagnostics prioritaires sont l'œdème aigu cardiogénique (cardiopathie connue, clinique [galop], peptide natriurétique de type B (BNP), échographie cardiaque ou test diurétique avec du furosémide non-source connu d'œdème contrairement à l'hydrochlorothiazide) et l'infection. L'objectif est de ne pas prendre de retard à la mise en route des traitements anti-infectieux notamment en cas d'aplasie. Il est des situations permettant d'évoquer d'emblée un diagnostic de PM comme la survenue d'une IRA alors que le patient est en cours de perfusion (tocolyse chez une femme enceinte, transfusion, chimiothérapie...) ou présente une triade évocatrice d'hémorragie intra-alvéolaire (hémoptysie, infiltrat, anémie) avec des anticorps anticytoplasme des polynucléaires neutrophiles présents chez un patient sous propylthiouracile.

À ce stade, soit les diagnostics prioritaires paraissent satisfaisants pour écarter le diagnostic d'atteinte respiratoire iatrogène, soit le diagnostic est incertain et les hypothèses alternatives rares dont le diagnostic de PM sont soulevées : localisation de la maladie sous-jacente et en milieu oncohématologique : protéinose alvéolaire en cas d'hémopathies myéloïdes notamment, œdème lésionnel par leucostase ou lyse, et pneumopathie radique. Le diagnostic d'hémorragie intra-alvéolaire liée aux troubles de coagulation doit rester un diagnostic d'exclusion.

\section{Réalisation d'examens complémentaires}

Les examens suivants sont discutés en fonction de la balance bénéfice/risque : fibroscopie bronchique (FB) avec un lavage bronchoalvéolaire (LBA), scanner thoracique et biopsie pulmonaire (Fig. 1).

\section{FB avec le $L B A$}

Le LBA permet de porter aisément un certain nombre de diagnostics : hémorragie intra-alvéolaire patente, pneumopathie aiguë à éosinophile, infection (notamment opportuniste), en évitant ainsi le recours à la réalisation d'un scanner thoracique ou d'une biopsie pulmonaire. Le LBA comprendra une analyse macroscopique, cytologique et microbiologique indispensable pour ne pas méconnaître une cause infectieuse. Il peut exceptionnellement apporter un diagnostic de certitude avec la mise en évidence de talc ou de silicone, mais plus souvent circonscrire la discussion diagnostique :

- les alvéolites lymphocytaires rapportées avec de nombreux traitements (méthotrexate, bévacizumab, sirolimus...) $[15,44]$ font discuter les pneumopathies d'hypersensibilité, les collagénoses, les infections à Chlamydia pneumoniae ou Mycoplasma pneumoniae et la tuberculose. Un chiffre très élevé de lymphocytes, leur nature $\mathrm{CD} 8+$ ou la présence associée de mastocytes plaident en faveur de l'hypersensibilité :

- les alvéolites à polynucléaires éosinophiles $[45,46]$ s'observent au cours des pneumopathies liées aux agents antiinfectieux [47], à certains anti-inflammatoires stéroïdiens et à la carbamazépine. Le contexte clinique et les examens complémentaires orientés écartent les parasitoses et les vascularites ; 


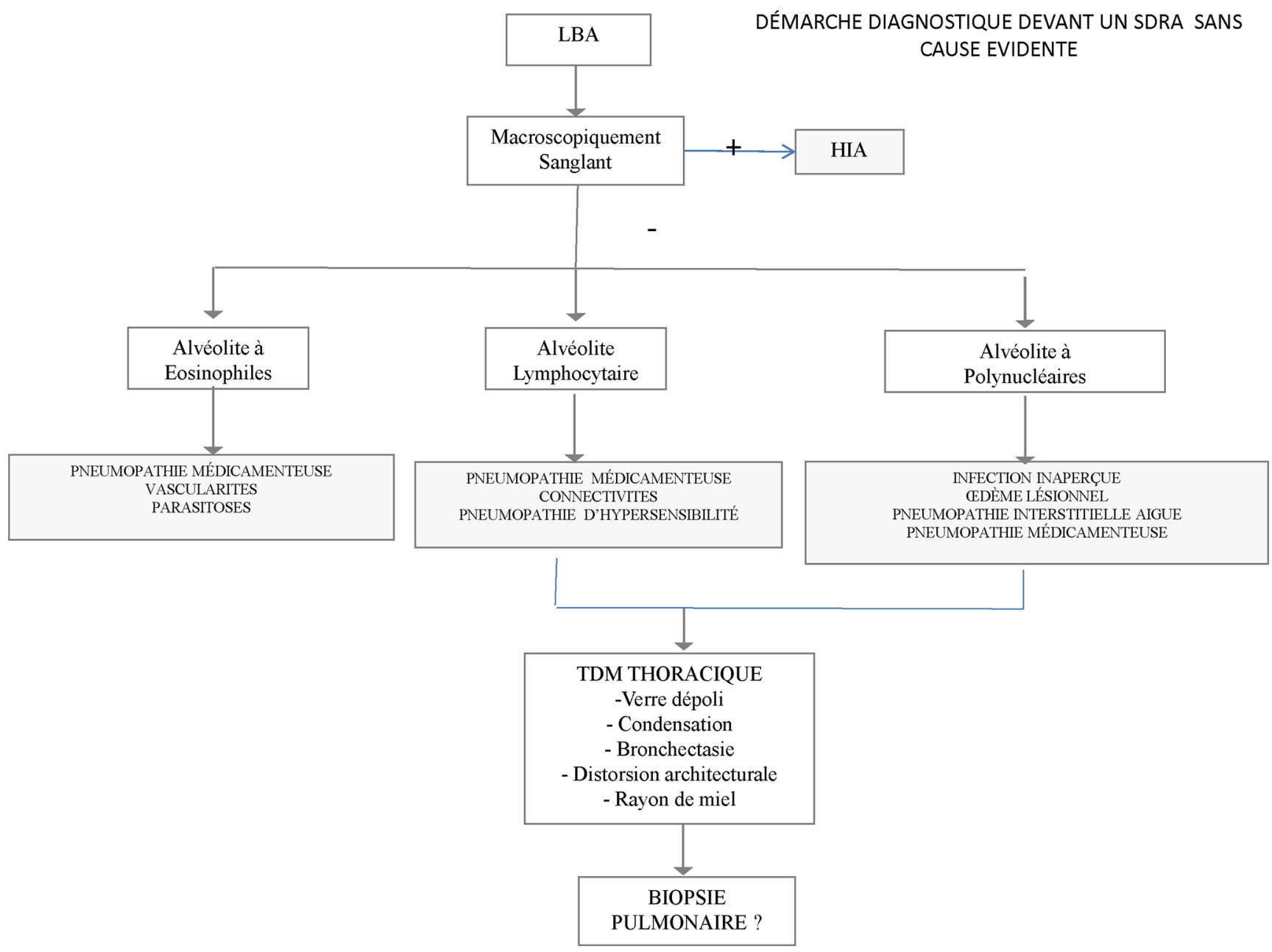

Fig. 1 Démarche diagnostique devant un SDRA sans cause évidente

- les alvéolites à polynucléaires neutrophiles [48] s'observent au cours des œdèmes lésionnels et des pneumopathies fibrosantes aiguës, quelle que soit leur origine, médicamenteuse ou autre. L'infection représente le diagnostic différentiel à ne pas méconnaître ;

- les alvéolites panachées [49], loin d'être exceptionnelles, recoupent les problèmes diagnostiques propres à chaque type d'alvéolite.

En pratique, chez le patient en neutropénie profonde, la probabilité d'une origine médicamenteuse et les modifications thérapeutiques induites par les résultats du LBA sont faibles [50]. Le traitement probabiliste associant antibiotiques et antifongiques et correction des désordres de l'hémostase est privilégié. La FB avec LBA n'est réalisée qu'en cas d'atypie clinique initiale ou en cours d'évolution, ou chez les patients traités par stéroïdes [51]. Chez les patients non neutropéniques, la FB avec LBA est largement discutée en soulignant le risque de recours à la ventilation invasive chez environ $10 \%$ des patients.

\section{Scanner thoracique}

Le scanner thoracique en haute résolution est indispensable, en dehors des situations où le LBA fournit un diagnostic indiscutable (hémorragie intra-alvéolaire patente, pneumopathie aiguë à éosinophile), car il permet une analyse sémiologique fine (plages d'hyperdensité en verre dépoli, condensation alvéolaire, bronchectasies de traction, signes de distorsion architecturale, image en rayon de miel) et de l'étendue des lésions [52,53]. Il peut aider à une classification des complications pulmonaires des traitements en suggérant le processus histopathologique, en mettant en évidence un aspect prédominant en faveur d'un dommage alvéolaire diffus (Fig. 2), d'une pneumonie interstitielle non spécifique (Fig. 3), d'une pneumonie organisée, d'une pneumopathie à éosinophile (Fig. 4) ou des patterns mixtes $[2,13,54]$ avec une bonne concordance interobservateur [24]. Cette classification aurait un intérêt pronostique, avec une mortalité significativement plus élevée en cas d'aspect de dommage alvéolaire diffus [2]. 


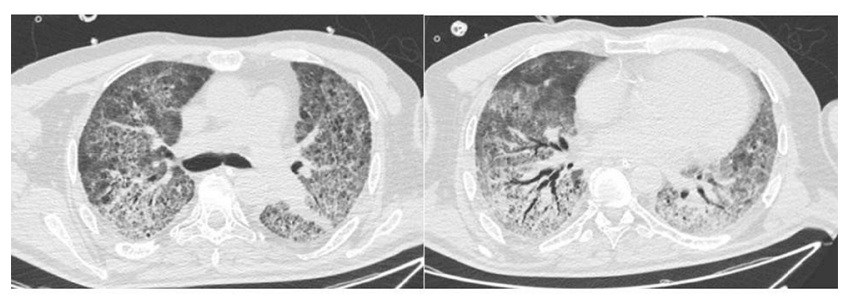

Fig. 2 Dommage alvéolaire diffus induit par la cordarone. Homme de 68 ans, suivi pour une fibrillation auriculaire traitée par amiodarone depuis quatre mois. Détresse respiratoire installée une semaine nécessitant l'optiflow puis intubation. LBA : contamination bronchique à prédominance de macrophages. TDM thoracique : verre dépoli, réticulations fines, bronchectasies par traction, prédominance périhilaire et basale. Évolution : décès malgré corticothérapie et cyclophosphamide

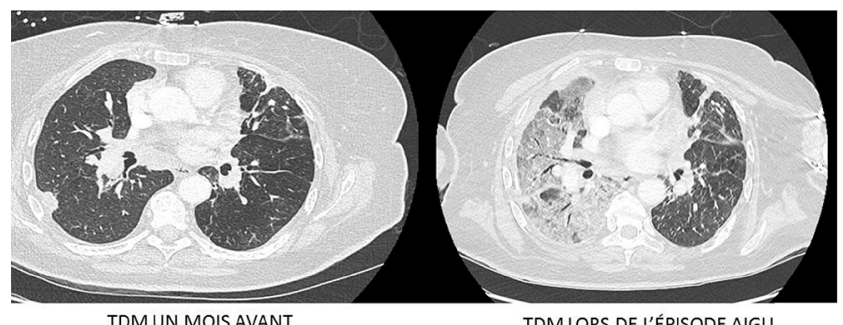

Fig. 3 Pneumopathie interstitielle non spécifique au brigatinib. Femme de 67 ans, suivie pour un cancer pulmonaire multimétastatique découvert depuis deux mois et traité par brigatinib. Détresse respiratoire installée en cinq jours nécessitant l'optiflow puis l'intubation à $\mathrm{j} 3$. LBA : 200000 cellules $/ \mathrm{mm}^{3}$ dont $33 \%$ de macrophages, $59 \%$ de PNN, $5 \%$ de lymphocytes et $3 \%$ d'éosinophiles. TDM : globale stabilité de la masse lobaire supérieure droite de $50 \times 40 \mathrm{~mm}$. Apparition d'un infiltrat en verre dépoli diffus de l'ensemble de l'hémichamp pulmonaire droit avec plage de condensation postérobasale du lobe inférieur gauche. Évolution : favorable à l'arrêt médicament et sous corticoïdes

Dans la pratique, la qualité des images peut être décevante, car le patient est polypnéique ou sous ventilation invasive. Enfin, le scanner thoracique permet de guider les prélèvements, si une biopsie pulmonaire est envisagée par exemple.

\section{Histologie pulmonaire}

Elle n'est pas essentielle au diagnostic, car il n'y a pas d'aspect histologique spécifique, qu'un même médicament peut être associé à plusieurs aspects histologiques [27] bien décrit avec l'amiodarone [55], parfois même au sein du même prélèvement $[41,56]$. Elle peut montrer un aspect de dommage alvéolaire diffus [41], de pneumonie interstitielle non spéci-

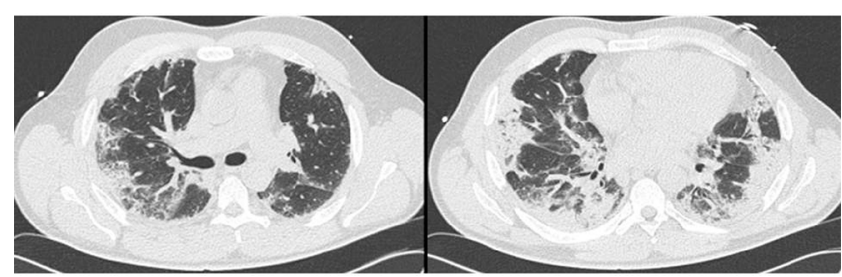

Fig. 4 Pneumopathie à éosinophile induite par la simvastatine. Homme de 47 ans, suivi pour une hypercholestérolémie traitée par simvastatine depuis trois mois. Détresse respiratoire $(9 \mathrm{l} / \mathrm{min}$ $\mathrm{d}^{\prime} \mathrm{O}_{2}$ ) avec une toux existante depuis un mois. Absence d'hyperéosinophilie sanguine. LBA : $56 \%$ de polynucléaires éosinophiles. TDM thoracique : condensation alvéolaire périphérique (OAP inversé) et opacités en verre dépoli. Évolution : favorable à l'arrêt médicament et sous corticoïdes

fique, de pneumonie organisée [42] et fibrineuse aiguë rapportée avec l'amiodarone [57,58], de pneumonie interstitielle desquamative, de pneumonie granulomateuse non nécrosante [59], ou d'une fibrose associée à une atteinte inflammatoire sans description précise. L'absence de macrophages spumeux plaidera éventuellement contre une pathologie à l'amiodarone [55].

La biopsie pulmonaire ne doit être réalisée qu'après un échec diagnostique de la FB et du LBA dans une situation d'impasse diagnostique pouvant conduire à un autre traitement que les corticoïdes, telle une chimiothérapie. Elle peut être obtenue par biopsie transbronchique, mais les prélèvements sont de petite taille et il existe un risque de pneumothorax suffocant dans cette situation d'IRA. La technique des cryobiopsies n'est pas adaptée aux patients en IRA. Le plus souvent, on a recours à une biopsie pulmonaire sous vidéothoracoscopie ou minithoracotomie, avec si possible une biopsie dans deux lobes en cas de lésions diffuses. Là encore, il faut évaluer les bénéfices et les risques, comparativement à l'administration d'une corticothérapie « à l'aveugle » [60], bien que les séries récentes rapportent une performance diagnostique et un impact thérapeutique intéressants de la biopsie pulmonaire, au prix d'une faible morbidité et mortalité (moins de $10 \%$ ). La biopsie pulmonaire doit être discutée au cas par cas sur des patients ayant peu de comorbidités (score de Charlson) et peu de défaillances d'organes, et réalisée précocement pour être la plus informative. L'analyse histologique requiert une expertise certaine, d'autant plus qu'il peut exister une grande variabilité interobservateur si l'on se rapporte à l'expérience des pneumopathies interstitielles diffuses. Dans notre expérience, quand la biopsie paraît indispensable, celle-ci peut être réalisée sous technique d'assistance extracorporelle à haut débit pour les cas les plus graves (SDRA). 


\section{Synthèse susceptible d'étayer l'hypothèse médicamenteuse ?}

En matière de pneumopathie médicamenteuse, on ne dispose pas de test diagnostique formel : les tests immunologiques dits spécifiques sont de sensibilité et de spécificité très discutées; le test de réintroduction n'est pas envisageable dans le contexte de forme asphyxiante. De toute façon, l'apport de ces tests ne serait que rétrospectif et peu contributif dans ces situations d'IRA. Par conséquent, le diagnostic ne peut être porté que par la démarche clinique en réunissant les éléments suivants :

- absence de diagnostic alternatif satisfaisant;

- établissement pour chaque médicament d'un score d'imputabilité intrinsèque, fondé sur des critères chronologiques (survenue des signes après l'instauration du médicament, notion de signes respiratoires lors de l'administration antérieure du médicament ou après une réintroduction accidentelle, piège de la chronologie = délai de survenue retardée, atteinte pulmonaire survenant après l'arrêt du traitement) et des critères sémiologiques respiratoires (comportant les données de l'examen physique, du scanner thoracique et du LBA [certains profils]) et extrarespiratoires (fièvre, rash, éosinophilie, et en cas d'immunothérapie atteinte d'autres organes : côlon, thyroïde, foie...) [24] ;

- recherche pour chaque médicament du score d'imputabilité extrinsèque, fondée sur les données bibliographiques de pneumotox (fréquence des effets secondaires pulmonaires rapportés, expression clinique de ces effets).

En pratique, soit le médicament a une forte réputation pneumotoxique (imputabilité extrinsèque forte) et le diagnostic est rapidement évoqué (par exemple amiodarone), mais il y a un risque de méconnaître une infection, soit le médicament a une faible réputation pneumotoxique (voire inconnue), et le risque est alors de méconnaître le diagnostic. Par le croisement de ces critères, on peut calculer pour chaque médicament le score global d'imputabilité, qui peut être incompatible, douteux, compatible, suggestif, très suggestif, pour limiter les erreurs par défaut comme par excès [61].

\section{Quel traitement?}

Le traitement symptomatique de la PM n'a pas de spécificité. Si la ventilation mécanique devient nécessaire, elle devra limiter le barotraumatisme et les hauts niveaux de $\mathrm{FiO}_{2}$, si cela ne s'avère pas nécessaire comme en cas de pneumopathie à la bléomycine [27]. Certains ont eu recours aux techniques d'assistance extracorporelle à haut débit.

La base du traitement est l'arrêt définitif du médicament incriminé [3]. Dans de rares cas, comme les œdèmes aux tocolytiques ou l'hypertension artérielle pulmonaire au dasatinib, cette simple mesure permet une évolution favorable.

La corticothérapie, dont les modalités d'administration (délai d'initiation, dose et durée) sont mal codifiées, est fré- quemment administrée, même si la preuve de son efficacité n'a jamais été démontrée par des essais contrôlés [2,16]. Ainsi, des améliorations, avec ou sans fibrose séquellaire et des décès, sous ou sans corticoïdes, sont rapportés. L'évolution de ces IRAM est en réalité difficilement prévisible ; dans notre expérience, le recours à la corticothérapie est fréquent avec une efficacité d'autant plus importante que son initiation est précoce [16]. Dans la littérature, notamment japonaise, des fortes doses (500 mgr-1 gr j1-j3 avec un relais à $1 \mathrm{mgr} / \mathrm{kg}$ par jour) sont rapportées, en tout cas, recommandées en cas d'hémorragie intra-alvéolaire [36]. Nous utilisons plutôt de fortes doses les trois premiers jours puis nous diminuons à $1 \mathrm{mgr} / \mathrm{kg}$ avec ensuite une décroissance d'autant plus lente que le médicament a une longue demi-vie ; ainsi pour l'amiodarone la durée de traitement pourra être de six mois. Enfin, l'adjonction d'immunosuppresseur tel que le cyclophosphamide et/ou l'infliximab ou les échanges plasmatiques sont à discuter selon la cause [24]. Exceptionnellement, des techniques d'hémoperfusion sur membrane de polymyxine B sont utilisées.

Le plus souvent, l'évolution est favorable avec une régression rapide pour des traitements tels que le méthotrexate et les antibiotiques, mais plus lente avec l'amiodarone. Parfois, il reste des séquelles fibrosantes comme avec la bléomycine ou au cours des pneumopathies radiques. Des rechutes à la diminution rapide d'une corticothérapie qui n'est pas codifiée à ce jour sont possibles.

Ultérieurement, pourra se poser l'épineuse question de la réintroduction en l'absence d'alternative qui peut être vitale en cas de cancer; dans certains cas, elle est possible sous une couverture éventuelle par corticoïdes [19].

\section{Conclusion}

Pour le réanimateur, les atteintes bronchopulmonaires médicamenteuses constituent un piège, source de deux types d'erreurs : une erreur par défaut, avec mise en place d'un traitement symptomatique de l'IRA tout en poursuivant le médicament responsable, et une erreur par excès, avec arrêt du médicament supposé responsable tout en méconnaissant la véritable cause de l'IRA. En pratique, il faut savoir évoquer le diagnostic, mais aussi l'étayer avec le maximum de rigueur.

Liens d'intérêt : Les auteurs déclarent ne pas avoir de lien d'intérêt.

\section{Références}

1. Jolivot PA, Hindlet P, Pichereau C, Fernandez C, Maury E, Guidet B, Hejblum G, (2014) A systematic review of adult admissions to ICUs related to adverse drug events. Crit care 18: 643 
2. Kim S, Oh IJ, Park SY, Song JH, Seon HJ, Kim YH, Yoon SH, Yu JY, Lee BR, Kim KS, Kim YC, (2014) Corticosteroid therapy against treatment-related pulmonary toxicities in patients with lung cancer. J Thorac Dis 6: 1209-1217

3. Vahid B, Marik PE, (2008) Pulmonary complications of novel antineoplastic agents for solid tumors. Chest 133: 528-538

4. Wu TY, Jen MH, Bottle A, Molokhia M, Aylin P, Bell D, Majeed A, (2010) Ten-year trends in hospital admissions for adverse drug reactions in England 1999-2009. J R Soc Med 103: 239-250

5. Dhokarh R, Li G, Schmickl CN, Kashyap R, Assudani J, Limper AH, Gajic O, (2012) Drug-associated acute lung injury: a population-based cohort study. Chest 142: $845-850$

6. Gibelin A, Parrot A, Maitre B, Brun-Buisson C, Mekontso Dessap A, Fartoukh M, de Prost N, (2016) Acute respiratory distress syndrome mimickers lacking common risk factors of the Berlin definition. Intensive Care Med 42: 164-172

7. Vial-Dupuy A, Sanchez O, Douvry B, Guetta L, Juvin K, Wermert D, Guérot E, Israël-Biet D, (2013) Outcome of patients with interstitial lung disease admitted to the intensive care unit. Sarcoidosis Vasc Diffuse Lung Dis 30: 134-142

8. Zafrani L, Lemiale V, Lapidus N, Lorillon G, Schlemmer B, Azoulay E, (2014) Acute respiratory failure in critically ill patients with interstitial lung disease. PloS One 9: e104897

9. Yoneda KY, Scranton JR, Cadogan MA, Tassell V, Nadanaciva S, Wilner KD, Stollenwerk NS, (2017) Interstitial lung disease associated with crizotinib in patients with advanced non-small cell lung cancer: independent review of four PROFILE trials. Clin Lung Cancer 18:472-9

10. Barber NA, Ganti AK, (2011) Pulmonary toxicities from targeted therapies: a review. Target Oncol 6: 235-243

11. Montani D, Seferian A, Savale L, Simonneau G, Humbert M, (2013) Drug-induced pulmonary arterial hypertension: a recent outbreak. Eur Respir Rev 22: 244-250

12. Nishino M, Giobbie-Hurder A, Hatabu H, Ramaiya NH, Hodi FS, (2016) Incidence of programmed cell death 1 inhibitorrelated pneumonitis in patients with advanced cancer: a systematic review and meta-analysis. JAMA Oncol 2: 1607-1616

13. Ramos-Casals M, Perez-Alvarez R, Perez-de-Lis M, Xaubet A, Bosch X, Group BS, (2011) Pulmonary disorders induced by monoclonal antibodies in patients with rheumatologic autoimmune diseases. Am J Med 124: 386-394

14. Teuwen LA, Van den Mooter T, Dirix L, (2015) Management of pulmonary toxicity associated with targeted anticancer therapies. Expert Opin Drug Metab Toxicol 11: 1695-1707

15. White DA, Camus P, Endo M, Escudier B, Calvo E, Akaza H, Uemura H, Kpamegan E, Kay A, Robson M, Ravaud A, Motzer RJ, (2010) Noninfectious pneumonitis after everolimus therapy for advanced renal cell carcinoma. Am J Respir Crit Care Med 182: 396-403

16. Lioté H, Cadranel J, Lioté F, (2010) Rituximab-induced pneumonitis: steroid therapy or not? Pediatr Pulmonol 45: 412-413

17. Gounant V, Brosseau S, Naltet C, Opsomer MA, Antoine M, Danel C, Khalil A, Cadranel J, Zalcman G, (2016) Nivolumabinduced organizing pneumonitis in a patient with lung sarcomatoid carcinoma. Lung cancer 99: 162-165

18. Nishino M, Sholl LM, Hodi FS, Hatabu H, Ramaiya NH, (2015) Anti-PD-1-related pneumonitis during cancer immunotherapy. N Engl J Med 373: 288-290

19. Kashiwabara K, Semba H, Fujii S, Tsumura S, (2017) Outcome in advanced non-small cell lung cancer patients with successful rechallenge after recovery from epidermal growth factor receptor tyrosine kinase inhibitor-induced interstitial lung disease. Cancer Chemother Pharmacol 79: 705-710

20. Bergeron A, Rea D, Levy V, Picard C, Meignin V, Tamburini J, Bruzzoni-Giovanelli H, Calvo F, Tazi A, Rousselot P, (2007) Lung abnormalities after dasatinib treatment for chronic myeloid leukemia: a case series. Am J Respir Crit Care Med 176: 814-818

21. Satoh T, Gemma A, Kudoh S, Sakai F, Yamaguchi K, Watanabe T, Ishiguro M, Inoshiri S, Izawa M, Sugihara K, Sakata Y, (2014) Incidence and Clinical Features of Drug-induced Lung Injury in Patients with Advanced Colorectal Cancer Receiving Cetuximab: Results of a Prospective Multicenter Registry. Jpn J Clin Oncol 44: 1032-1039

22. Johkoh T, Sakai F, Kusumoto M, Arakawa H, Harada R, Ueda M, Kudoh S, Fukuoka M (2014) Association between baseline pulmonary status and interstitial lung disease in patients with non-small-cell lung cancer treated with erlotinib-a cohort study. Clin Lung Cancer 15: 448-454

23. Shah RR, (2016) Tyrosine kinase inhibitor-induced interstitial lung disease: clinical features, diagnostic challenges, and therapeutic dilemmas. Drug Saf 39: 1073-1091

24. Naidoo J, Wang X, Woo KM, Iyriboz T, Halpenny D, Cunningham J, Chaft JE, Segal NH, Callahan MK, Lesokhin AM, Rosenberg J, Voss MH, Rudin CM, Rizvi H, Hou X, Rodriguez K, Albano M, Gordon RA, Leduc C, Rekhtman N, Harris B, Menzies AM, Guminski AD, Carlino MS, Kong BY, Wolchok JD, Postow MA, Long GV, Hellmann MD, (2017) Pneumonitis in patients treated with anti-programmed death-1/programmed death ligand 1 Therapy. J Clin Oncol 35: 709-717

25. Till BG, Madtes DK, (2012) BCNU-associated pneumonitis: portrait of a toxicity. Leuk Lymphoma 53: 1019-1020

26. Delaunay M, Cadranel J, Lusque A, Meyer N, Gounaut V, MoroSibilot D, Michot JM, Raimbourg J, Girard N, Guisier F, Planchard D, Metivier AC, Tomasini P, Dansin E, Pérol M, Campana M, Gautschi O, Früh M, Fumet JD, Audigier-Valette C, Couraud S, Dalle S, Leccia MT, Jaffro M, Collot S, Prévot G, Milia J, Mazieres $\mathrm{J}$, (2017) Immune-checkpoint inhibitors associated with interstitial lung disease in cancer patients. Eur Respir J (in press)

27. Matsuno O, (2012) Drug-induced interstitial lung disease: mechanisms and best diagnostic approaches. Respir Res 13: 39

28. Harada C, Kawaguchi T, Ogata-Suetsugu S, Yamada M, Hamada N, Maeyama T, Souzaki R, Tajiri T, Taguchi T, Kuwano K, Nakanishi Y, (2011) EGFR tyrosine kinase inhibition worsens acute lung injury in mice with repairing airway epithelium. Am J Respir Crit Care Med 183: 743-751

29. Culp JA, Palis RI, Castells MC, Lucas SR, Borish L, (2007) Perioperative anaphylaxis in a 44-year-old man. Allergy Asthma Proc 28: 602-605

30. Johnson DH, Fehrenbacher L, Novotny WF, Herbst RS, Nemunaitis JJ, Jablons DM, Langer CJ, DeVore RF $3^{\text {rd }}$, Gaudreault J, Damico LA, Holmgren E, Kabbinavar F, (2004) Randomized phase II trial comparing bevacizumab plus carboplatin and paclitaxel with carboplatin and paclitaxel alone in previously untreated locally advanced or metastatic non-small-cell lung cancer. J Clin Oncol 22: 2184-2191

31. Montani D, Bergot E, Günther S, Savale L, Bergeron A, Bourdin A, Bouvaist H, Canuet M, Pison C, Macro M, Poubeau P, Girerd B, Natali D, Guignabert C, Perros F, O'Callaghan DS, Jaïs X, Tubert-Bitter P, Zalcman G, Sitbon O, Simonneau G, Humbert M, (2012) Pulmonary arterial hypertension in patients treated by dasatinib. Circulation 125: 2128-2137

32. Ranchoux B, Günther S, Quarck R, Chaumais MC, Dorfmüller P, Antigny F, Dumas SJ, Raymond N, Lau E, Savale L, Jaïs X, Sitbon O, Simonneau G, Stenmark K, Cohen-Kaminsky S, Humbert M, Montani D, Perros F, (2015) Chemotherapy-induced pulmonary hypertension: role of alkylating agents. Am J Pathol 185: 356-371

33. Turco C, Jary M, Kim S, Moltenis M, Degano B, Manzoni P, Nguyen T, Genet B, Rabier MB, Heyd B, Borg C, (2015) Gemcitabine-induced pulmonary toxicity: a case report of pulmonary veno-occlusive disease. Clin Med Insights Oncol 9: 75-79 
34. Ieki R, Saitoh E, Shibuya M, (2003) Acute lung injury as a possible adverse drug reaction related to gefitinib. Eur Respir J 22: 179-181

35. Inoue A, Saijo Y, Maemondo M, Gomi K, Tokue Y, Kimura Y, Ebina M, Kikuchi T, Moriya T, Nukiwa T, (2003) Severe acute interstitial pneumonia and gefitinib. Lancet 361: 137-139

36. Créquit P, Wislez M, Fleury Feith J, Rozensztajn N, Jabot L, Friard S, Lavole A, Gounant V, Fillon J, Antoine M, Cadranel J, (2015) Crizotinib associated with ground-glass opacity predominant pattern interstitial lung disease: a retrospective observational cohort study with a systematic literature review. J Thorac Oncol 10: 1148-1155

37. Tamiya A, Okamoto I, Miyazaki M, Shimizu S, Kitaichi M, Nakagawa K, (2013) Severe acute interstitial lung disease after crizotinib therapy in a patient with EML4-ALK-positive nonsmall-cell lung cancer. J Clin Oncol 31: e15-e17

38. Otoshi T, Kataoka Y, Nakagawa A, Otsuka K, Tomii K, (2016) Clinical features and outcomes of diffuse alveolar hemorrhage during antithrombotic therapy: a retrospective cohort study. Lung 194: 475-481

39. Kurimoto R, Sekine I, Iwasawa S, Sakaida E, Tada Y, Tatsumi K, Takahashi Y, Nakatani Y, Imai C, Takiguchi Y, (2015) Alveolar hemorrhage associated with pemetrexed administration. Intern Med 54: 833-836

40. Ono A, Takahashi T, Oishi T, Sugino T, Akamatsu H, Shukuya T, Taira T, Kenmotsu H, Naito T, Murakami H, Nakajima T, Endo M, Yamamoto N, (2013) Acute lung injury with alveolar hemorrhage as adverse drug reaction related to crizotinib. J Clin Oncol 31: e417-e419

41. Lioté H, Lioté F, Seroussi B, Mayaud C, Cadranel J, (2010) Rituximab-induced lung disease: A systematic literature review. Eur Respir J 35: 681-687

42. Barjaktarevic IZ, Qadir N, Suri A, Santamauro JT, Stover D, (2013) Organizing pneumonia as a side effect of ipilimumab treatment of melanoma. Chest 143: 858-861

43. Mankikian J, Lioger B, Diot E, D'Halluin P, Lissandre S, Marchand Adam S, Mercier E, Beau Salinas F, (2014) Pulmonary toxicity associated with the use of lenalidomide: case report of late-onset acute respiratory distress syndrome and literature review. Heart Lung 43: 120-123

44. Fuhrman C, Parrot A, Wislez M, Prigent H, Boussaud V, Bernaudin JF, Mayaud C, Cadranel J, (2001) Spectrum of CD4 to CD8 T-cell ratios in lymphocytic alveolitis associated with methotrexateinduced pneumonitis. Am J Respir Crit Care Med 164: 1186-1191

45. Souza CA, Muller NL, Johkoh T, Akira M, (2006) Drug-induced eosinophilic pneumonia: high-resolution CT findings in 14 patients. AJR Am J Roentgenol 186: 368-373

46. St John RC, Allen JN, Pacht ER, (1990) Postoperative respiratory failure due to acute eosinophilic pneumonia. Intensive Care Med 16: $408-410$
47. Griffiths CL, Gutierrez KC, Pitt RD, Lovell RD, (2014) Eosinophilic pneumonia induced by ceftaroline. Am J Health Syst Pharm 71: 403-406

48. Salmeron S, Brochard L, Rain B, Herve P, Brenot F, Simonneau G, Duroux P, (1988) Early neutrophil alveolitis after rechallenge in drug induced alveolitis. Thorax 43: 647-648

49. Khalil H, Molinary E, Stoller JK, (1993) Diclofenac (Voltaren)induced eosinophilic pneumonitis. Case report and review of the literature. Arch Intern Med 153: 1649-1652

50. Gruson D, Hilbert G, Valentino R, Vargas F, Chene G, Bebear C, Allery A, Pigneux A, Gbikpi-Benissan G, Cardinaud JP, (2000) Utility of fiberoptic bronchoscopy in neutropenic patients admitted to the intensive care unit with pulmonary infiltrates. Crit Care Med 28: 2224-2230

51. Cordonnier C, Escudier E, Verra F, Brochard L, Bernaudin JF, Fleury-Feith J, (1994) Bronchoalveolar lavage during neutropenic episodes: diagnostic yield and cellular pattern. Eur Respir J 7: 114-120

52. Endo M, Johkoh T, Kimura K, Yamamoto N, (2006) Imaging of gefitinib-related interstitial lung disease: multi-institutional analysis by the West Japan Thoracic Oncology Group. Lung cancer 52: $135-140$

53. Torrisi JM, Schwartz LH, Gollub MJ, Ginsberg MS, Bosl GJ, Hricak H, (2011) CT findings of chemotherapy-induced toxicity: what radiologists need to know about the clinical and radiologic manifestations of chemotherapy toxicity. Radiology 258: 41-56

54. Rossi SE, Erasmus JJ, McAdams HP, Sporn TA, Goodman PC, (2000) Pulmonary drug toxicity: radiologic and pathologic manifestations. Radiographics 20: 1245-1259

55. Larsen BT, Vaszar LT, Colby TV, Tazelaar HD, (2012) Lymphoid hyperplasia and eosinophilic pneumonia as histologic manifestations of amiodarone-induced lung toxicity. Am J Surg Pathology 36: $509-516$

56. Feenstra JF, Hickey BP, Blackwell EA, (2004) Acute respiratory failure associated with cladribine pneumonitis. Intern Med J 34: 583-584

57. Beasley MB, Franks TJ, Galvin JR, Gochuico B, Travis WD, (2002) Acute fibrinous and organizing pneumonia: a histological pattern of lung injury and possible variant of diffuse alveolar damage. Arch Pathol Lab Med 126: 1064-1070

58. Piciucchi S, Dubini A, Tomassetti S, Casoni G, Ravaglia C, Poletti V, (2015) A case of amiodarone-induced acute fibrinous and organizing pneumonia mimicking mesothelioma. Am J Respir Crit Care Med 191: 104-106

59. Helman DL, Jr., Byrd JC, Ales NC, Shorr AF, (2002) Fludarabinerelated pulmonary toxicity: a distinct clinical entity in chronic lymphoproliferative syndromes. Chest 122: 785-790

60. Schwarz MI, Albert RK, (2004) "Imitators" of the ARDS: implications for diagnosis and treatment. Chest 125: 1530-1535

61. Lioté H, Seroussi B, Bouaud J, Voiriot G, Mayaud C, (2007) PneumoDoc: a computer-based decision-making system for drug-related pulmonary disease. Rev Pneumol Clin 63: 193-201 